\title{
Osteonecrose dos maxilares relacionada ao uso de medicamentos: revisão sistemática
}

\author{
Osteonecrosis of the jaws related to medicinal use: systematic review \\ Osteonecrosis de los maxilares relacionada com el uso de medicamentos: revisión sistemática
}

Recebido: 06/04/2021 | Revisado: 13/04/2021 | Aceito: 17/04/2021 | Publicado: 27/04/2021

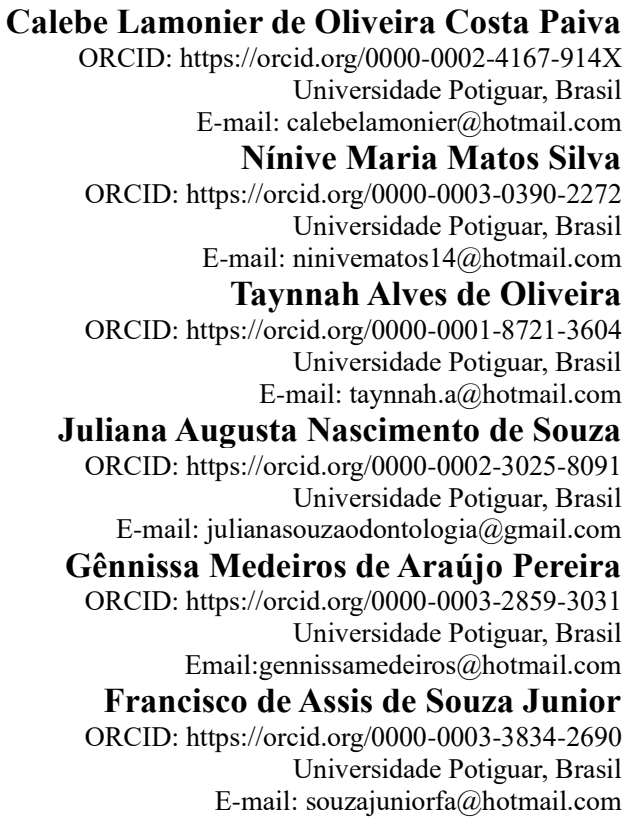

\begin{abstract}
Resumo
A osteonecrose dos maxilares relacionada ao uso de medicamentos (OMRM) é uma situação clínica proveniente de efeitos secundários da terapêutica com alguns fármacos, como os bifosfonatos, os inibidores do RANK-L e alguns antiangiogênicos. O objetivo do presente trabalho foi realizar uma revisão sistemática sobre OMRM. As pesquisas realizadas permitiram a seleção de 9 artigos, a partir de 759 artigos encontrados. Os resultados do presente estudo mostram que a doença subjacente mais comum foi o câncer de mama, a via de administração mais prevalente foi a intravenosa, extração dentária foi o principal procedimento odontológico desencadeador e a região mais acometida pela alteração foi a mandíbula. $\mathrm{O}$ atendimento pelo cirurgião-dentista se torna fundamental antes e durante a terapia com os medicamentos estudados, no intuito de promover saúde ao sistema estomatognático e diminuir os riscos de complicações maxilofaciais.
\end{abstract}

Palavras-chave: Osteonecrose; Bifosfonatos; Mandíbula; Procedimentos Cirúrgicos Bucais; Maxila.

\begin{abstract}
Medication-related osteonecrosis of the jaw (MRONJ) is a clinical situation resulting from side effects of therapy with some drugs, such as bisphosphonates, RANK-L inhibitors and some antiangiogenics. The objective of the present work was to carry out a systematic review on OMRM. The research carried out allowed the selection of 9 articles, from 759 articles found. The results of the present study show that the most common underlying disease was breast cancer, the most prevalent route of administration was intravenous, tooth extraction was the main triggering dental procedure and the region most affected by the change was the mandible. The attendance by the dentist becomes essential before and during the therapy with the studied drugs, in order to promote health to the stomatognathic system and to reduce the risks of maxillofacial complications.
\end{abstract}

Keywords: Osteonecrosis; Diphosphonates; Mandible; Oral Surgical Procedures; Maxilla.

\section{Resumen}

La osteonecrosis de los maxilares relacionada con el uso de medicamentos (OMRM) es una situación clínica resultante de los efectos secundarios de la terapia con algunos medicamentos, como los bisfosfonatos, los inhibidores de RANK-L y algunos antiangiogénicos. El objetivo del presente trabajo fue realizar una revisión sistemática sobre OMRM. La investigación realizada permitió la selección de 9 artículos, de 759 artículos encontrados. Los resultados del presente estudio muestran que la enfermedad subyacente más común fue el cáncer de mama, la vía de 
administración más prevalente fue la intravenosa, la extracción dentaria fue el principal procedimiento desencadenante y la región más afectada por el cambio fue la mandíbula. La asistencia del odontólogo se vuelve imprescindible antes y durante la terapia con los fármacos estudiados, con el fin de promover la salud del sistema estomatognático y reducir los riesgos de complicaciones maxilofaciales.

Palabras clave: Osteonecrosis; Difosfonatos; Mandíbula; Procedimientos Quirúrgicos Orales; Maxilar.

\section{Introdução}

A osteonecrose dos maxilares relacionada ao uso de medicamentos (OMRM) é uma situação clínica relativamente rara, mas potencialmente grave e debilitante, proveniente de efeitos secundários da terapêutica com alguns fármacos, sendo eles os bifosfonatos, os inibidores do RANK-L como o denosumab, e alguns antiangiogénicos como o bevacizumabe. Os bifosfonatos são medicamentos análogos sintéticos de pirofosfato inorgânico, um composto naturalmente presente no organismo e um regulador fisiológico da calcificação e inibidor da reabsorção óssea (Eid, \& Atlas, 2014). Os bifosfonatos são drogas que suprimem a reabsorção óssea, sendo a terapia de primeira escolha no tratamento de doenças do metabolismo dos ossos (osteoporose e doença de paget) e malignidades envolvendo osso. Eles diminuem a função dos osteoclastos e, portanto, a reabsorção óssea, estabilizando o processo osteoporótico, o crescimento adicional e formação de metástases no osso, assim melhorando queixas, tais como dor (Di Fede et al., 2018; Pichardo, Kuypers, \& van Merkesteyn, 2013).

Desde 1977 os bifosfonatos têm sido utilizados como tratamento para a osteoporose nos Estados Unidos. Entretanto, somente em 2003 que Marx fez a primeiro relato do termo "osteonecrose dos maxilares" (do inglês "osteonecrosis of the jaws" ou ONJ) apresentando 36 casos de exposições ósseas dolorosas, em mandíbula e/ou maxila, que pouco responsivo ao tratamento médico ou cirúrgico, em pacientes que utilizavam pamidronato e ácido zolendrônico (Wilkinson, Kuo, Freeman, \&Goodwin, 2007; Louraço, 2012; Vilela-Carvalho, Tuany-Duarte, Andrade-Figueiredo, \& López-Ortega, 2018).

O denosumab é um inibidor de RANK-L que é usado no tratamento de distúrbios ósseos, como a osteoporose e a metástase óssea de tumores malignos. Ao impedir a ligação do RANKL com o RANK, inibe a atividade osteoclástica e, consequentemente, dificulta a reabsorção óssea, aumenta a densidade óssea e redução do risco de fratura. (Ribeiro et al., 2018). O receptor ativador nuclear карра B (RANK), ligante do receptor ativador nuclear каppa B (RANKL) e osteoprotegerina (OPG) são membros da família do fator de necrose tumoral relacionados com o metabolismo ósseo. A formação, diferenciação e atividade dos osteoclastos são reguladas por estas três proteínas. RANK é um receptor presente em vários tipos celulares, principalmente em células de linhagem macrofágica, linfócitos, células dendríticas e fibroblastos, que quando ativado pelo seu ligante, RANKL, promove a ativação de células osteoclásticas (Moraes, 2010). Os fármacos antiangiogênicos podem comprometer a integridade de microvasos por dificultar a ação do VEGF (fator de crescimento do endotélio vascular), o que pode ter efeitos deletérios sobre a diferenciação celular e a função óssea e, dessa forma, induzir a OMRM (Ribeiroet al., 2018).

Há muitas hipóteses sobre patogênese OMRM, mas nenhuma delas é completamente aceita (Zhang et al., 2013). O objetivo do presente trabalho foi realizar uma revisão sistemática sobre OMRM em busca de elucidar quais procedimentos desencadeadores estariam relacionados com o surgimento da alteração e quais tratamentos poderiam ser realizados nesses casos, bem como identificação de medidas de prevenção para esta condição.

\section{Metodologia}

Trata-se de uma revisão sistemática de literatura. A revisão sistemática consiste em uma revisão planejada para responder a uma pergunta, utilizando métodos acessíveis e sistemáticos para identificar, selecionar e avaliar de forma crítica os estudos, coletando e analisando os dados oriundos dos estudos existentes na revisão (Castro, A. A., 2001).

\subsection{Critérios de elegibilidade}


A questão focal foi estabelecida de acordo com o formato PICO (população, intervenção, comparação, desfecho): Em pacientes com neoplasias malignas ou doenças ósseas (P), submetidos a terapia com bifosfonato, antiangiogênicos ou inibidores RANK-L (I), que desenvolveram osteonecrose dos maxilares (C), tiveram qual evento desencadeador e tratamento realizado $(\mathrm{O})$ ?

- P (População): pacientes com neoplasias malignas ou doenças ósseas;

- I (Intervenção): submetidos a terapia com bifosfonato, antiangiogênicos ou inibidores RANK-L

- C (Comparação): desenvolveram osteonecrose dos maxilares;

- $\quad$ O (Desfecho): evento desencadeador e tratamento realizado.

Os critérios de inclusão e exclusão estão listados no quadro 1.

Quadro 1: Fatores de inclusão e exclusão.

\begin{tabular}{|l|ll|}
\hline \multicolumn{1}{|c|}{ Fatores de Inclusão } & Fatores de Exclusão \\
\hline a. $\quad \begin{array}{l}\text { Ensaios clínicos randomizados, ensaios clínicos } \\
\text { controlados e estudos de coorte prospectivos, } \\
\text { estudos prospectivos e retrospectivos com ou } \\
\text { sem grupo controle. }\end{array}$ & 1. Revisões da literatura \\
\hline b. $\quad$ Amostra com, no mínimo, 10 pacientes. & 3. Carta ao editor (crítica do leitor). \\
\hline c. $\quad$ Estudos publicados nos últimos 10 anos. & 4. Artigos que não abordem a temática proposta. \\
\hline d. $\quad$ Estudos em inglês, espanhol e português &
\end{tabular}

Fonte: Autores, (2021).

\subsection{Fontes de informação}

Foram utilizados 07 (sete) bases de dados, sendo elas a PubMed, ScienteDirect, Scopus, Biblioteca Virtual em SaúdeBVS, Hindawi, Cochrane e Google Acadêmico, para realizar as buscar bibliográficas. A última busca realizada foi em 15 de abril de 2021.

\subsection{Busca}

Foram criados 04 (quatro) grupos de palavras com os descritores para busca, sendo o grupo 01 relacionado ao nome da patologia, o 02 e 03 envolvendo as medicações que possivelmente desencadeiam a alteração e o grupo 04 com as áreas da odontologia que atuam realizando procedimentos que tem forte potencial de desenvolvimento da OMRM. Os operadores booleanos utilizados estão descritos no quadro 2. Todos os descritores utilizados foram pesquisados em três idiomas, sendo eles inglês, espanhol e português. Os descritores utilizados nas buscas se encontram cadastrados no DeCS (Descritores em Ciências da Saúde). O refinamento da busca foi realizado através da limitação em "últimos 10 anos", baseado no critério de inclusão C, quadro 1.

Quadro 2: Grupos de descritores. Os mesmos descritores também foram utilizados em espanhol e português.

\begin{tabular}{|l|l|}
\hline Grupos & Descritores \\
\hline $\mathbf{0 1}$ & $\begin{array}{l}\text { (Osteonecrosis) AND (Mandible) AND (Maxilla) AND ("Bisphosphonate-Associated Osteonecrosis } \\
\text { of the Jaw") AND (Diphosphonates OR Bisphosphonates) }\end{array}$ \\
\hline $\mathbf{0 2}$ & (Bevacizumab) AND (Denosumab) AND (Osteonecrosis) AND (jaw) \\
\hline $\mathbf{0 3}$ & (Osteonecrosis) AND (Diphosphonates OR Bisphosphonates) AND (EtidronicAcid) AND \\
\hline
\end{tabular}




\begin{tabular}{|l|l|}
\hline & (Alendronate) AND ("RisedronicAcid") AND ("IbandronicAcid”) AND ("ZoledronicAcid") \\
\hline $\mathbf{0 4}$ & $\begin{array}{l}\text { (Osteonecrosis) AND (“Dental Implants") AND (Endodontics) AND (Periodontics) AND ("Dental } \\
\text { Prosthesis") AND ("Oral Surgical Procedures") }\end{array}$ \\
\hline
\end{tabular}

Fonte: Autores, (2021).

\subsection{Processo de coleta de dados}

As buscas pelos estudos foram realizadas independentemente por dois revisores previamente calibrados por um terceiro revisor. Todos os títulos e resumos de trabalhos avaliados como elegíveis foram separados e analisados completamente. A seleção dos artigos ocorreu inicialmente pela leitura de títulos, depois leitura de resumos e leitura completa de artigos, aplicando os critérios de inclusão e exclusão (Quadro 1).

\subsection{Lista dos dados}

As informações extraídas inicialmente de cada estudo foram organizadas em uma tabela do programa Excel 2012, obtendo e registrando as seguintes informações para organização e exploração pelos autores: Título; Ano de publicação, Autor(es); Base de dados e Link de acesso.

Fluxograma 1: Fluxograma da revisão sistemática.
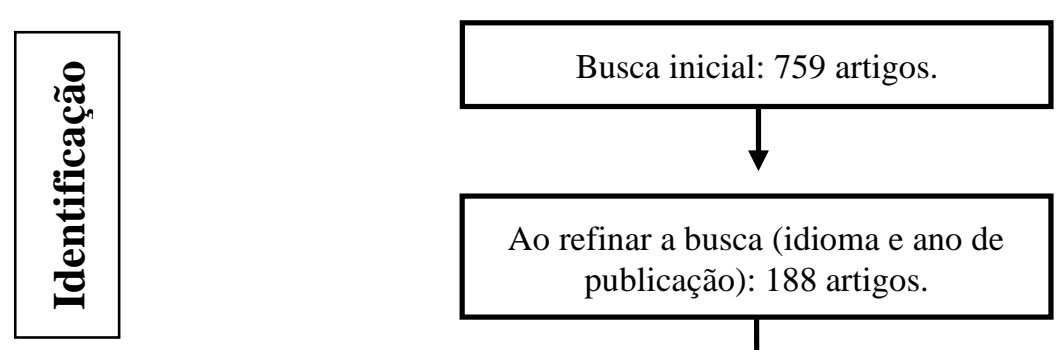

Ao refinar a busca (idioma e ano de publicação): 188 artigos.
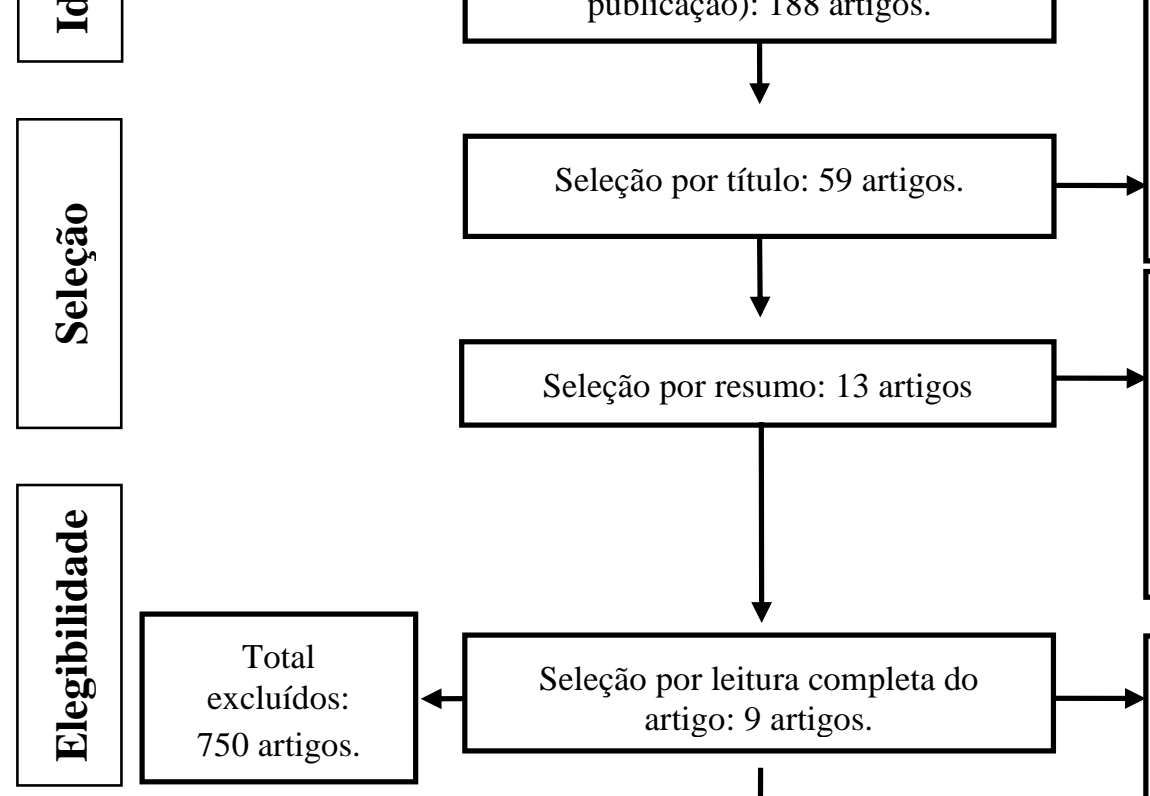

Excluídos por leitura de título: 129 artigos.

* Justificativas:

- 62 excluídos pelo item 4*.

- 52 pelo item $1^{*}$.

- 29 pelo item $2^{*}$.

- 4 pelo item $3^{*}$.

Excluídos por leitura do resumo: 46

* Justificativas:

- 21 pelo item $4 *$.

- 2 pelo item $2 *$.

- 10 pelo item $1 *$

- 13 repetidos
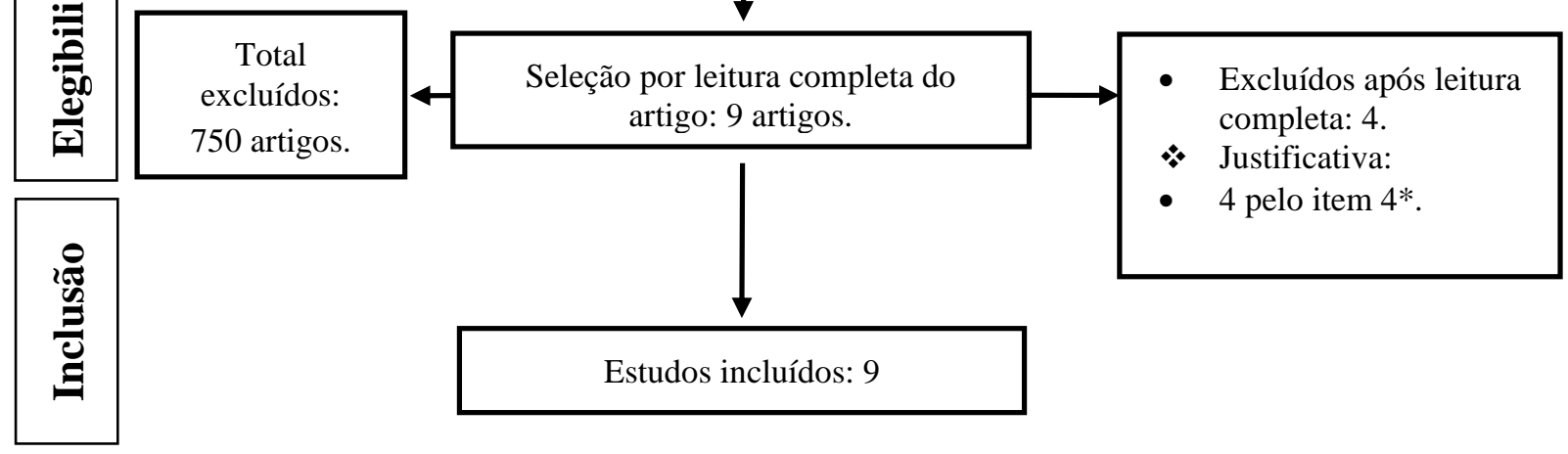

* Conferir o item no Quadro 1 de critérios de inclusão e exclusão.

Fonte: Autores, (2021). 


\section{Resultados}

\subsection{Seleção de estudos}

As pesquisas realizadas nas bases de dados com a metodologia já descrita, resultou no encontro de 759 artigos. Estratégias de busca, refinamento e uma revisão detalhada dos títulos, resumos e leitura completa dos artigos, utilizando critérios de inclusão e exclusão (Quadro 1) permitiu a seleção de 9 artigos, com um total de 750 artigos excluídos (Fluxograma 1).

Figura 1: Ano de publicação dos estudos elegíveis.

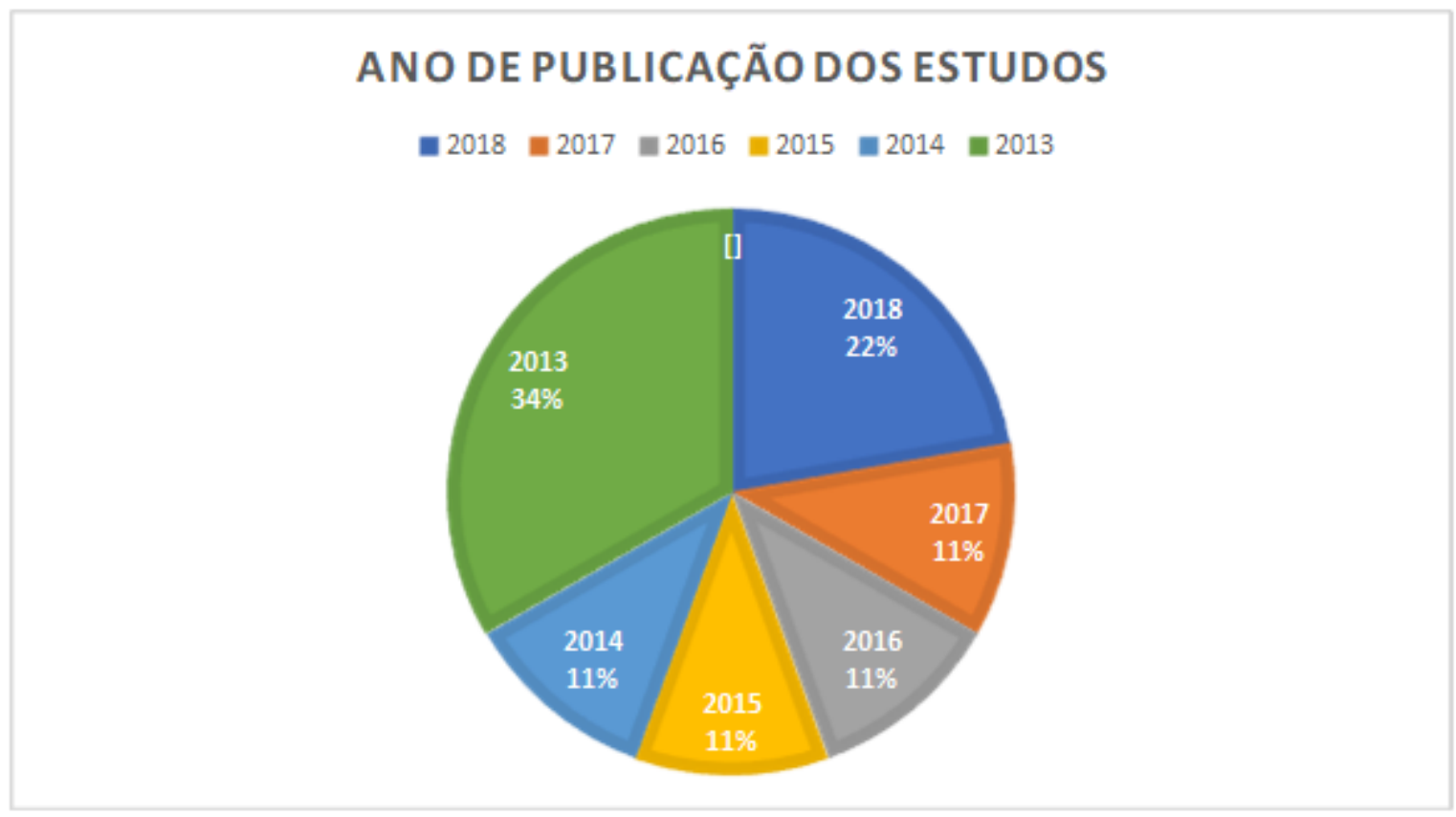

Fonte: Autores, (2021).

\subsection{Informações dos estudos e doença subjacente}

O ano de publicação mais frequente entre os estudos selecionados foi 2013 (Figura 1). A doença subjacente mais relacionada ao surgimento da OMRM foi o câncer de mama, presente em 7 dos 9 estudos avaliados.

Quadro 3: Informações dos estudos e doença subjacente.

\begin{tabular}{|c|c|c|c|}
\hline AUTORES & ANO & $\begin{array}{c}\text { TIPO DE ESTUDO / AMOSTRA } \\
\text { (No CASOS) }\end{array}$ & DOENÇA SUBJACENTE \\
\hline 01. Qizhang Wang, et al. & 2018 & Estudos de casos / 201 casos. & Câncer de mama \\
\hline 02. Al-Rabadi H, et al. & 2018 & Estudo retrospectivo / 12 casos. & $\begin{array}{l}\text { Mieloma múltiplo, câncer de mama, } \\
\text { câncer de próstata e osteoporose }\end{array}$ \\
\hline 03. Leticia Bagan, et al. & 2017 & Estudos de casos / 183 casos. & Câncer de mama, mieloma múltiplo \\
\hline 04. Gianfranco Favia, et al. & 2016 & Estudo coorte / 244 casos. & Osteoporose, câncer de mama e mieloma \\
\hline 05. Carmen Vidal-Real, et al. & 2015 & Relatos de casos / 194 casos. & $\begin{array}{l}\text { Câncer de próstata, câncer de mama e } \\
\text { mieloma }\end{array}$ \\
\hline 06. Bedogni Alberto, et al. & 2014 & Estudos de casos / 545 casos. & Câncer de mama e Mieloma \\
\hline
\end{tabular}




\begin{tabular}{|c|c|c|c|}
\hline 07. E. Mercer, et al. & 2013 & Relatos de casos / 91 casos. & Osteoporose \\
\hline 08. Olga Di Fede, et al. & 2013 & Estudos de casos / 87 casos. & Osteoporose \\
\hline 09. Daniel Holzinger, et al. & 2013 & Estudos de casos / 108 casos. & $\begin{array}{l}\text { Mieloma múltiplo, câncer de mama e } \\
\text { osteoporose. }\end{array}$ \\
\hline
\end{tabular}

Fonte: Autores, (2021).

\subsection{Medicamento, via de administração, dose, duração e procedimento desencadeador}

A medicação mais utilizada conforme os estudos foi o ácido zoledrônico, tendo como via de administração a via Intravenosa. Em segundo lugar vem o aledronato, tendo como via de administração utilizada a via oral. O tempo de uso do medicamento não foi informado pela maioria dos estudos. O procedimento desencadeador da OMRM mais comum foi a extração dentária.

Quadro 4: Medicamento, via de administração, dose, duração e procedimento desencadeador.

\begin{tabular}{|c|c|c|c|}
\hline ESTUDO & $\begin{array}{l}\text { MEDICAMENTO / VIA DE } \\
\text { ADMINISTRAÇÃO }\end{array}$ & $\begin{array}{c}\text { DOSE / TEMPO DE } \\
\text { USO }\end{array}$ & DESENCADEADOR \\
\hline 01. Qizhang Wang, et al. (2018) & $\begin{array}{l}\text { Ácido Zoledrônico / } \\
\text { Intravenoso }\end{array}$ & Não informado & Extração dentária \\
\hline 02. Al-Rabadi H, et al. (2018) & $\begin{array}{l}\text { Pamidronato e Ácido } \\
\text { Zoledrônico / Intravenoso }\end{array}$ & Não informado / 34 meses & Extração dentária \\
\hline 03. Leticia Bagan, et al. (2017) & $\begin{array}{l}\text { Ácido Zoledrônico / } \\
\text { Intravenoso }\end{array}$ & Não informado & Extração dentária \\
\hline $\begin{array}{l}\text { 04. Gianfranco Favia, et al. } \\
\text { (2016) }\end{array}$ & $\begin{array}{l}\text { Ácido Zoledrônico / } \\
\text { Intravenoso }\end{array}$ & Não informado & Não informado \\
\hline $\begin{array}{l}\text { 05. Carmen Vidal-Real, et al. } \\
\text { (2015) }\end{array}$ & $\begin{array}{l}\text { Ácido Zoledrônico / } \\
\text { Intravenoso }\end{array}$ & $\begin{array}{l}\text { Doses de } 4 \mathrm{mg} \text { com } \\
\text { intervalos mensais } / 7 \\
\text { anos }\end{array}$ & Extração dentária \\
\hline 06. Bedogni Alberto, et al. (2014) & Pamidronato / Intravenoso & Não informado & Extração dentária \\
\hline 07. E. Mercer, et al. (2013) & Alendronato / Via oral & Não informado / 5 anos & $\begin{array}{l}\text { Extração dentária e implante } \\
\text { dentário }\end{array}$ \\
\hline 08. Olga Di Fede, et al. (2013) & Alendronato / Via oral & $\begin{array}{l}\text { Não informado / } 2 \text { meses } \\
\text { a } 16 \text { anos }\end{array}$ & Extração dentária \\
\hline $\begin{array}{l}\text { 09. Daniel Holzinger, et al. } \\
\text { (2013) }\end{array}$ & Aledronato / Via oral & Não informado & Espontâneo \\
\hline
\end{tabular}

Fonte: Autores, (2021).

\subsection{Localização da lesão, tratamento e tempo de tratamento}

A idade de acometimento pela OMRM teve uma ampla faixa de variação, sendo constatado casos a partir de 29 anos até 94 anos, porém, uma incidência maior entre 64 a 72 anos. A localização mais comum foi mandíbula, predominando em todos os estudos analisados. O tratamento mais empregado foi cirúrgico. 
Quadro 5: Localização, tratamento e tempo de tratamento.

\begin{tabular}{|c|c|c|c|c|}
\hline ESTUDO & IDADE / SEXO & $\begin{array}{c}\text { LOCALIZAÇÃO } \\
\text { DA LESÃO }\end{array}$ & TRATAMENTO & $\begin{array}{c}\text { TEMPO DE } \\
\text { TRATAMENTO }\end{array}$ \\
\hline $\begin{array}{l}\text { 01. Qizhang Wang, } \\
\text { et al. (2018) }\end{array}$ & Em média 64,43 / F & Mandíbula & $\begin{array}{lr}\text { Tratamento } & \text { cirúrgico } \\
\text { (sequestrectomia, } & \text { curetagem } \\
\text { profunda, ressecção segmentar) }\end{array}$ & Não informado \\
\hline $\begin{array}{l}\text { 02. Al-Rabadi H, } \\
\text { et al. (2018) }\end{array}$ & Em média 62 / F & Mandíbula & Intervenções cirúrgicas & 4 a 8 semanas \\
\hline $\begin{array}{l}\text { 03. Leticia Bagan, } \\
\text { et al. (2017) }\end{array}$ & Em média 68,22 / F & Mandíbula & Não informado & Não informado \\
\hline $\begin{array}{l}\text { 04. Gianfranco } \\
\text { Favia, et al. } \\
\text { (2016) }\end{array}$ & Não informado/ F & Mandíbula & Abordagens cirúrgicas & Não informado \\
\hline $\begin{array}{l}\text { 05. Carmen Vidal- } \\
\text { Real, et al. } \\
(2015)\end{array}$ & $\begin{array}{l}\text { Em média } 68,91 / \\
\mathrm{M}\end{array}$ & Mandíbula & Não informado & Não informado \\
\hline $\begin{array}{l}\text { 06. Bedogni } \\
\text { Alberto, et al. } \\
\text { (2014) }\end{array}$ & Em média 61,5 / M & Mandíbula & Não informado & Não informado \\
\hline $\begin{array}{l}\text { 07. E. Mercer, et } \\
\text { al. (2013) }\end{array}$ & Em média 71 / F & Mandíbula & Intervenções cirúrgicas & Não informado \\
\hline $\begin{array}{l}\text { 08. Olga Di Fede, } \\
\text { et al. (2013) }\end{array}$ & $\begin{array}{l}\text { Em média } 72 \text { anos / } \\
F\end{array}$ & Mandíbula & Não informado & Não informado \\
\hline $\begin{array}{l}\text { 09. Daniel } \\
\text { Holzinger, et } \\
\text { al. (2013) }\end{array}$ & Não informado/ F & Mandíbula & Intervenções cirúrgicas & Não informado \\
\hline
\end{tabular}

Fonte: Autores, (2021).

\section{Discussão}

A OMRM é uma condição patológica relativamente incomum, com os primeiros relatos em 2003. (Marx, R.E. 2003) Centenas de publicações relacionada ao tema foram publicados posteriormente, envolvendo outras categorias medicamentosas não relatadas incialmente por Marx. Essa alteração é de grande relevância a saúde humana, visto que está relacionado a efeitos secundários de medicações muito utilizadas pela população, como exemplo os bifosfonatos. Menos frequentemente, também está ligado ao uso de outras medicações, são eles: inibidores do RANK-L como o denosumab, e alguns antiangiogênicos como o bevacizumabe. O uso dessas medicações associado a execução de procedimentos cirúrgicos orais pode levar ao surgimento da osteonecrose. Apesar de incomum, o presente estudo detectou que a OMRM também pode acontecer de forma espontânea, citado em 1 dos 9 estudos analisados.

Em relação a doença subjacente mais comum entre casos diagnosticados com OMRM, foi o câncer de mama, porém, outras doenças como osteoporose, mieloma múltiplo e câncer de próstata também foram relacionadas. O medicamento mais relacionado a osteonecrose foi o ácido zoledrônico. Essa forte relação do ácido zoledrônico com o surgimento da 
OMRM é descrita na literatura no estudo de brozoski et. al., na qual complementa que são necessárias aproximadamente seis doses mensais de ácido zoledrônico endovenoso para que o paciente apresente risco de desenvolver OMRM. (Brozoskiet al., 2012; Berti-Couto et al., 2014; Ruggiero, \& Woo, 2008)

No tocante ao procedimento que mais tem desencadeado a OMRM temos a extração dentária como a mais relatada, concordando com o estudo de Barrier et. al. que apresentou maior número de casos de osteonecrose nos maxilares ocorrendo após procedimentos invasivos como exodontia e o com o estudo de Sedghizadeh et al. na qual todos os casos ocorreram após exodontias. (Barrieret al., 2010; Sedghizadeh et al., 2009). Isso pode ser justificado pelo fato da exodontia ser um dos procedimentos cirúrgicos orais mais realizados pelos profissionais. Em relação ao sexo de predileção, o feminino apresentou maior percentual, com 77,77\%, em uma proporção 7:2 dos estudos avaliados. A idade média de acometimento foi de 66,86 anos, mostrando maior associação com idosos. Uma possível explicação pode ser que pessoas mais velhas são mais propensas a desenvolver patologias ósseas como osteoporose ou doenças malignas, sendo muitas vezes prescrito o uso de bifosfonatos para esses casos. (Qizhang Wang, et al., 2018)

A região mais acometida pela alteração foi a mandíbula, representando $100 \%$ dos estudos ( 9 dos 9 estudos), que possivelmente relacionado está relacionado com menor vascularização comparada ao osso maxilar. Entretanto, no estudo realizado por Qizhang Wang, et al., mostrou que 36,67\% das lesões de OMRM ocorreram na maxila e na série de casos realizada por Dimitrakopoulos et al. encontrou uma incidência maior na maxila em comparação com a mandíbula, ambos estudos contrastando com o presente estudo. É importante enfatizar que o acometimento maxilar apresenta o risco de levar a sintomas graves, como sinusite maxilar, perfuração do seio maxilar, sendo propenso a piores prognósticos. (Qizhang Wang, et al., 2018; Dimitrakopoulos I, Magopoulos C, Karakasis D., 2006). O acometimento de osteonecrose nos ossos maxilares (maxila e mandíbula) se deve ao maior suprimento sanguíneo e um metabolismo ósseo acelerado que esses ossos possuem. Devido às suas atividades fisiológicas diárias e a constante remodelação óssea envolta do ligamento periodontal, gera uma maior concentração destas drogas nos ossos maxilares. (Lopes I; Zenha H; Costa H, Barroso J., 2009; Ruggieroet al., 2009)

Sobre o tratamento, o presente estudo mostra que abordagens cirúrgicas resultam na maioria dos casos (constatandose $45 \%$ dos estudos) que foram avaliados, dentre essas abordagens temos desbridamento cirúrgico, ressecção cirúrgica e reconstrução e sequestrectomia. A AAOMS (Associação Americana de Cirurgiões Orais e Maxilofaciais) desenvolveu estratégias de tratamento que deve ser indicado conforme o estadiamento da condição. Na categoria de risco (em uso de bifosfonato oral ou IV, sem osso necrótico aparente) a estratégia é orientações ao paciente quanto à possibilidade de desenvolvimento da doença, reforçando a necessidade de maiores cuidados preventivos. No estágio 0 (sem evidência de osso necrótico, mas com achados clínicos e sintomas não específicos) a abordagem seria o uso de fármacos como analgésicos e antibiótico. No estágio 1 (osso necrótico exposto sem infecção) a estratégia de tratamento é uso de bochechos antibacterianos, orientação e revisão das indicações de uso contínuo de bifosfonato. No estágio 2 (osso necrótico exposto com infecção) a estratégia é uso de antibiótico VO, bochecho antibacteriano, controle da dor e debridamento superficial. No estágio 3, além de osso necrótico exposto, dor e eritema, pode ter envolvimento além dos processos alveolares (borda inferior ou ramo mandibular, seio maxilar, zigomático, resultando em fratura patológica, fístula extraoral, comunicação bucossinual ou nasal), a estratégia de tratamento será por meio de antibiótico via oral, bochecho antibacteriano, controle da dor e debridamento ou ressecção. (Ruggieroet al., 2009)

Como prevenção, o teste CTx (telopeptídeo carboxi-terminal do colágeno tipo I, ou ICTP) pode ser utilizado para avaliar o risco de desenvolvimento da OMRM, visto que é um marcador de remodelação óssea usado para medir o nível da atividade metabólica do tecido ósseo, devendo o mesmo ter valor inferior a $150 \mathrm{pg} / \mathrm{mL}$ para realizar os procedimentos 
odontológicos. Embora existam recomendações para interromper este medicamento dois a três meses antes da cirurgia, há falta de evidências confirmando este protocolo, pois a suspensão não consiste em uma estratégia de redução de risco, visto que as medicações, principalmente os bifosfonatos, são medicamentos com ação residual na medida em que se ligam ao osso, permanecendo no tecido por anos. Diferentemente dos bifosfonatos, após suspensão, o denosumabe pode apresentar seus efeitos por um menor período de tempo, isso devido sua alta afinidade pelo RANK-L e a baixa relação com a hidroxiapatita, sendo relatado na literatura que seus efeitos se finalizem após 6 meses (Hellstein J., 2014; Hamadeh IS, Ngwa BA, Gong Y., 2015). Damn, \& Jones recomendaram que a medicação deve ser suspensa 2 meses antes da intervenção oral, visto que a quantidade de bifosfonatos livres seria muito baixa dois meses após a última dose (Damn, \& Jones, 2013). Já a AAOMS sugere que a pausa no uso da medicação seja de 3 meses antes do procedimento e 3 meses após a intervenção cirúrgica oral. Segundo Vilela-Carvalho LN, os pacientes que receberam cuidados odontológicos preventivos, antes de iniciar a terapia com drogas antiangiogênicas e antirresorptivas, tiveram uma diminuição de 50\% no risco de desenvolver a OMRM, atestando a importância da avaliação pelo cirurgião-dentista antes do início das terapias. (Brozoskiet al., 2012; Ruggiero et al., 2009; Vilela-Carvalho, Tuany-Duarte, Andrade-Figueiredo, \& López-Ortega, 2018).

\section{Considerações Finais}

Diante de todas as medicações relacionadas à osteonecrose dos maxilares, o ácido zoledrônico (do grupo dos bifosfonatos) está intimamente associado ao surgimento da OMRM, a via de administração endovenosa/intravenosa aparenta ser de maior risco, a doença subjacente mais relacionada a OMRM nesse estudo foi o câncer de mama, porém, certamente o surgimento não esteja relacionado ao tipo de câncer, e sim ao tratamento medicamentoso empregado. Já em relação aos exames complementares, o CTx pode ser utilizado no auxílio da avaliação de risco de desenvolvimento de OMRM. O procedimento cirúrgico oral de extração dentária possui alto risco desencadeador da OMRM nos pacientes de risco (que faz uso das medicações relacionadas, mas sem sintomas). As intervenções em região mandibular devem ser bem ponderadas pelos cirurgiões-dentistas em relação ao risco e benefício, visto que é a região mais acometida. O tratamento cirúrgico tem desempenhado importante papel no tratamento da alteração, apresentando maiores chances de resolutividade, no entanto faz-se necessário mais estudos por modalidades terapêuticas menos invasivas. $\mathrm{O}$ atendimento pelo cirurgião-dentista se torna fundamental antes e durante a terapia com os medicamentos estudados, no intuito de promover saúde ao sistema estomatognático e diminuir os riscos de complicações maxilofaciais. Entretanto, as drogas avaliadas necessitam de mais estudos por melhorias farmacológicas, para que atuem de forma efetiva em sua finalidade clínica sem comprometer os ossos maxilares.

\section{Referências}

Eid, A., \& Atlas, J. (2014). The role of bisphosphonates in medical oncology and their association with jaw bone necrosis. Oral andMaxillofacialSurgeryClinics, 26(2), 231-237

Di Fede, O., Panzarella, V., Mauceri, R., Fusco, V., Bedogni, A., LoMuzio, L., ... \& Campisi, G. (2018). The dental management of patients at risk of medication-related osteonecrosis of the jaw: new paradigm of primary prevention. BioMedresearchinternational, 2018.

Pichardo, S. E., Kuypers, S. C., \& Van Merkesteyn, J. R. (2013). Denosumab osteonecrosis of the mandible: a new entity? A case report. Journal of CranioMaxillofacialSurgery, 41(4), e65-e69.

Wilkinson, G. S., Kuo, Y. F., Freeman, J. L., \& Goodwin, J. S. (2007). Intravenous bisphosphonate therapy and inflammatory conditions or surgery of the jaw: a population-based analysis. JournaloftheNationalCancerInstitute, 99(13), 1016-1024.

Louraço, A. M. C. (2012). Terapêutica com Bisfosfonatos e a colocação de Implantes: Revisão da Literatura (Doctoraldissertation).

Vilela-Carvalho, L. N., Tuany-Duarte, N., Andrade-Figueiredo, M., \& López-Ortega, K. (2018). Osteonecrose dos maxilares relacionada ao uso de medicações: Diagnóstico, tratamento e prevenção. CES Odontología, 31(2), 48-63. 
Ribeiro, G. H., Chrun, E. S., Dutra, K. L., Daniel, F. I., \& Grando, L. J. (2018). Osteonecrosis of the jaws: a review and update in etiology and treatment. Brazilianjournalofotorhinolaryngology, 84(1), 102-108

Moraes, M. D. (2010). Expressão imuno-hitoquímica das proteínas RANK, RANKL e OPG em cistos radiculares e cistos dentígeros (Master'sthesis, Universidade Federal do Rio Grande do Norte).

Ruggiero, S. L., Dodson, T. B., Assael, L. A., Landesberg, R., Marx, R. E., \& Mehrotra, B. (2009). American Association of Oral and Maxillofacial Surgeons position paper on bisphosphonate-related osteonecrosis of the jaws--2009 update. Journalof Oral andMaxillofacialSurgery, 67(5), 2-12.

Zhang, J., Xie, F., Delzell, E., Chen, L., Kilgore, M. L., Yun, H., ... \& Curtis, J. R. (2013). Trends in the use of biologic agents among rheumatoid arthritis patients enrolled in the US medicare program. Arthritiscare\&research, 65(11), 1743-1751.

Wang, Q., Liu, J.,Qi, S., Liao, X., Liu, D., \& Pan, J. (2018). Clinical analysis of medication related osteonecrosis of the jaws: A growing severe complication in China. Journalof dental sciences, 13(3), 190-197.

Al-Rabadi, H., Daklalah, L. K., Alwreikat, M., Alqudah, M., Momani, M., \& Nsour, H. F. (2018). Bisphosphonate Induced Osteonecrosis of the Jaws: Our Clinical Experience at King Hussein Medical Center, Amman, Jordan. The journalofcontemporary dental practice, 19(11), 1400-1403.

Bagan, L., Jiménez, Y., Leopoldo, M., Murillo-Cortes, J., \& Bagan, J. (2017). Exposed necrotic bone in 183 patients with bisphosphonate-related osteonecrosis of the jaw: Associated clinical characteristics. Medicina oral, patologia oral y cirugia bucal, 22(5), e582.

Favia, G., Tempesta, A., Limongelli, L., Crincoli, V., \& Maiorano, E. (2016). Medication-relatedosteonecrosisofthejaws: considerationson a new antiresorptivetherapy (Denosumab) andtreatmentoutcomeafter a 13-year experience. Internationaljournalofdentistry, 2016.

Vidal-Real, C., Pérez-Sayáns, M., Suárez-Peñaranda, J. M., Gándara-Rey, J. M., \& García-García, A. (2015). Osteonecrosisofthejaws in 194 patientswhohaveundergoneintravenousbisphosphonatetherapy in Spain. Medicina oral, patologia oral y cirugia bucal, 20 (3), e267.

Khan, A. A., Morrison, A., Hanley, D. A., Felsenberg, D., McCauley, L. K., O'Ryan, F., ... \& InternationalTask Force onOsteonecrosisoftheJaw. (2015). Diagnosisand management ofosteonecrosisofthejaw: a systematicreviewandinternational consensus. JournalofBoneand Mineral Research, $30(1), 3-23$.

Bedogni, A., Fedele, S., Bedogni, G., Scoletta, M., Favia, G., Colella, G., ...\& Campisi, G. (2014). Stagingofosteonecrosisofthejawrequirescomputedtomography for accuratedefinitionoftheextentofbonydisease. British Journalof Oral andMaxillofacialSurgery, 52(7), 603-608

Mercer, E., Norton, T., Woo, S., Treister, N., Dodson, T. B., \&Solomon, D. H. (2013). Ninety-oneosteoporosispatientsaffectedwithbisphosphonaterelatedosteonecrosisofthejaw: a case series. Calcifiedtissueinternational, 93(3), 241-248.

Holzinger, D., Seemann, R., Klug, C., Ewers, R., Millesi, G., Baumann, A., \& Wutzl, A. (2013). Long-termsuccessofsurgery in bisphosphonaterelatedosteonecrosisofthejaws (BRONJs). Oral oncology, 49(1), 66-70.

Brozoski, M. A., Traina, A. A., Deboni, M. C. Z., Marques, M. M., \& Naclério-Homem, M. D. G. (2012). Osteonecrose maxilar associada ao uso de bisfosfonatos. Revista Brasileira de reumatologia, 52(2), 265-270.

Marx, R. E. (2003). Pamidronate (Aredia) and zoledronate (Zometa) induced avascular necrosis of the jaws: a growing epidemic. Journal of Oral and Maxillofacial Surgery, 61(9), 1115-1117.

Berti-Couto, S. A., Vasconcelos, A. C. U., Iglesias, J. E., Figueiredo, M. A. Z., Salum, F. G., \& Cherubini, K. (2014). Diabetes mellitus andcorticotherapy as riskfactors for alendronate-relatedosteonecrosisofthejaws: a study in Wistarrats. Head \&neck, 36(1), 84-93.

Ruggiero, S. L., \& Woo, S. B. (2008). Biophosphonate-relatedosteonecrosisofthejaws. Dental Clinicsof North America, 52(1), 111-128.

Barrier, A., Lescaille, G., Rigolet, A., Descroix, V., Goudot, P., \& Ruhin, B. (2010). Jawosteonecrosisinducedby oral biphosphonates: 12 cases. Revue de Stomatologie et de ChirurgieMaxillo-faciale, 111(4), 196-202.

Sedghizadeh, P. P., Stanley, K.,Caligiuri, M., Hofkes, S., Lowry, B., \& Shuler, C. F. (2009). Oral bisphosphonate use andtheprevalenceofosteonecrosisofthejaw: aninstitutionalinquiry. The Journalofthe American Dental Association, 140(1), 61-66.

Dimitrakopoulos, I., Magopoulos, C., \& Karakasis, D. (2006). Bisphosphonate-induced avascular osteonecrosis of the jaws: a clinical report of 11 cases. International journal of oral and maxillofacial surgery, 35(7), 588-593.

Lopes, I., Zenha, H., Costa, H., \& Barroso, J. (2009). Osteonecrose da mandíbula associada ao uso de bifosfonatos: uma patologia secundária grave. Arquivos de Medicina, 23(5), 181-185.

Castro A A. (2001). Revisão sistemática e meta-aálise. Elaboração e Apresentação de Comunicação Científica. São Paulo.

Greenberg, M. S. (2004). Intravenousbisphosphonatesandosteonecrosis. Oral surgery, oral medicine, oral pathology, oral radiology, andendodontics, 98(3), $259-260$.

Van denWyngaert, T., Huizing, M. T., \&Vermorken, J. B. (2006). Bisphosphonatesandosteonecrosisofthejaw: cause andeffector a post hoc fallacy?. AnnalsofOncology, 17(8), 1197-1204

Morais, E. F., de Paiva Macedo, R. A., da Silva Lira, J. A., \& de Arruda, M. D. L. S. (2016). Osteonecrose dos maxilares associado ao uso de alendronato oral em paciente sob tratamento de osteoporose/Osteonecrosisjawassociatedwithalendronate for oral use in patientsunderosteoporosistreatment. CATUSSABAISSN 2237-3608, 5(1), 21-29.

Hellstein, J. (2014). Osteochemonecrosis: an overview. Head and neck pathology, 8(4), 482-490. 
Research, Society and Development, v. 10, n. 5, e15510514740, 2021

(CC BY 4.0) | ISSN 2525-3409 | DOI: http://dx.doi.org/10.33448/rsd-v10i5.14740

Hamadeh, I. S., Ngwa, B. A., \& Gong, Y. (2015). Drug induced osteonecrosis of the jaw. Cancer treatment reviews, $41(5), 455-464$.

Damm, D. D., \& Jones, D. M. (2013). Bisphosphonate-related osteonecrosis of the jaws: a potential alternative to drug holidays. GenDent, 61(5), 33-38. 